\title{
Surface Plasmon Radiation Forces
}

\author{
Giovanni Volpe, ${ }^{1}$ Romain Quidant, ${ }^{1,2}$ Gonçal Badenes, ${ }^{1}$ and Dmitri Petrov ${ }^{1,2, *}$ \\ ${ }^{1}$ ICFO - Institut de Ciències Fotòniques, 08860, Castelldefels (Barcelona), Spain \\ ${ }^{2}$ ICREA - Institució Catalana de Recerca i Estudis Avançats, 08010, Barcelona, Spain
}

(Received 7 November 2005; published 13 June 2006)

\begin{abstract}
We report the first experimental observation of momentum transfer from a surface plasmon to a single dielectric sphere. Using a photonic force microscope, we measure the plasmon radiation forces on different polystyrene beads as a function of their distance from the metal surface. We show that the force magnitude at resonance is strongly enhanced compared to a nonresonant illumination. Measurements performed as a function of the probe particle size indicate that optical manipulation by plasmon fields has a strong potential for optical sorting.
\end{abstract}

The momentum exchange between light waves and matter is a fundamental process that has been exploited for remarkable applications such as atom cooling [1] and optical tweezers $[2,3]$. Following recent advances in nanophotonics, optical manipulation by evanescent fields, instead of conventional propagating fields, has lately awoken an increasing interest $[4,5]$. The main motivations for using nonradiative fields are (i) the absence of the diffraction limit for the trapping volume, which may permit an effective manipulation of single subwavelength objects, and (ii) the intrinsic in-plane field confinement, which is of interest for lab-on-a-chip applications. Momentum transfer from evanescent fields to micro- and nanoparticles has been extensively investigated theoretically and experimentally both near the surface of a dielectric prism illuminated under total internal reflection and near to an optical waveguide [6-11].

In order to extend the range of in-plane optical manipulation it has lately been proposed to use surface plasmons (SP) $[4,12-14]$. SP are electromagnetic surface modes confined at a metal-dielectric interface due to the resonant interaction of the electromagnetic wave with the surface charges of the metal $[15,16]$. They give rise to a multifold increase of the transverse magnetic (TM)-polarized incident field that is expected to result in a significant enhancement of the radiation forces on a nearby object. Until now, SP forces exerted on $0.5 \mu \mathrm{m}$ diameter metal or dielectric spheres have only been studied theoretically [17]. For a particle far enough from the surface, such that it does not perturb the plasmon field, the radiation forces are predicted to increase considerably in the presence of SP.

We report in this Letter the first experimental observation of the momentum transfer from a SP to a single dielectric object. Using a photonic force microscope (PFM), we have quantitatively measured the plasmon radiation forces on polystyrene beads of various sizes, when located at different distances from the metal surface. We show that the magnitude of the force at resonance on a $4.5 \mu \mathrm{m}$ dielectric sphere is enhanced 40 times compared to nonresonant illumination.
The PFM uses a micron-sized particle optically trapped by a focused laser beam, as a local probe, whose position can be measured with great accuracy $[18,19]$. The analysis of the Brownian motion of this probe provides information on the local forces it experiences.

Our experimental setup, presented in Fig. 1, consists of three main parts: the sample, the SP illumination, and the optical tweezers and position detection (PFM).

The sample solution is prepared by adding a small amount of polystyrene beads of three sizes (diameter 4.5, 2.0 , and $0.6 \mu \mathrm{m}$, refractive index $n_{b}=1.59$ ) to a $10 \%$ sodium dodecyl sulphate sterile solution in water (refractive index $\left.n_{s}=1.33\right)$. A drop $(2 \mu \mathrm{l})$ of the resulting solution is placed between two coverslips (thickness $80 \mu \mathrm{m}$, refractive index $n_{c}=1.55$ ) separated by a $30 \mu \mathrm{m}$ spacer and sealed with water-insoluble silicone vacuum grease to prevent an evaporation. The upper cover slip is coated with

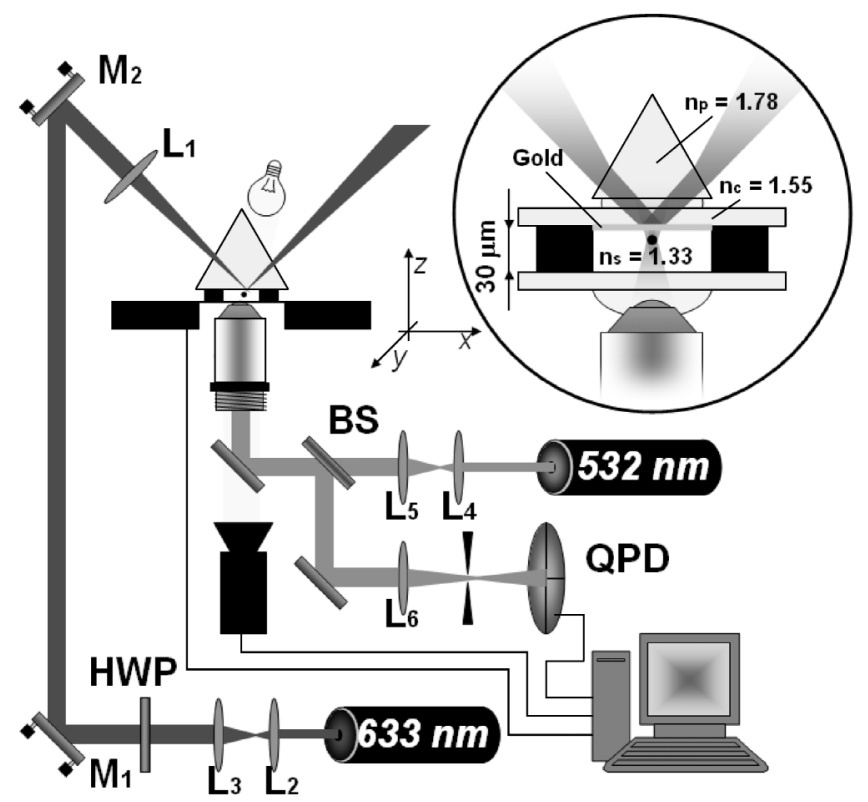

FIG. 1. Schematic of the experimental setup. Inset: close-up view of the chamber. 
a $40 \mathrm{~nm}$ layer of gold. This sample is placed onto a custommade sample holder between an oil immersion microscope objective $(\times 100, N A=1.3)$ and a prism $\left(n_{p}=1.78\right)$ to couple the SP. A piezoelectric stage (Tritor 102, Piezosystem Jena) is used for 3D-nanometer positioning of the sample relative to the probe.

A linearly polarized beam from a He-Ne laser (wavelength $632.8 \mathrm{~nm}$, power $18 \mathrm{~mW}$ ) illuminates the metalwater interface through the prism at the plasmon resonance angle $\left(\theta_{\mathrm{SP}}=70.8^{\circ}\right)$. Mirrors $M_{1}$ and $M_{2}$ allow fine adjustment of the incident angle, while a lens $\left(L_{1}\right.$, focal distance $5 \mathrm{~cm}$ ) focuses the beam to increase the intensity of the evanescent wave. A half wave plate (HWP) permits us to change the incident polarization between TM and TE. The resulting wave generated at the metal surface is expected from theory [15] to decay exponentially as $e^{-\beta z}$ with a penetration depth $d_{p}=\beta^{-1}=\frac{\lambda}{2 \pi\left[\left(n_{c} \sin \theta_{\mathrm{SP}}\right)^{2}-n_{s}^{2}\right]^{1 / 2}}=165 \mathrm{~nm}$, where $\lambda$ is the wavelength in vacuum.

A $532 \mathrm{~nm}$ laser beam, focused by the objective, traps the probe particle and positions it in close vicinity to the upper cover slip. The back-scattered light from the particle is collected by the same objective, passes through a series of filters and a beam splitter (BS) and is detected by a quadrant photo-diode (QPD) (Silicon Sensors, QP50-6-SD). We maintain a low-power ( $3 \mathrm{~mW}$ at the probe) of the trapping beam to achieve a high sensitivity of the position detection to external forces. The resulting sum and differential signals carry the information about the particle coordinates. This information, together with the knowledge of the temperature and the viscosity of the surrounding medium, allows us to reconstruct the optical trapping potential, and to calibrate the trap. For each cartesian coordinate the power spectral density (PSD) of the Brownian motion of a bead in an optical trap is given by $P(f)=\frac{D /\left(2 \pi^{2}\right)}{f_{c}^{2}+f^{2}}$, where $f_{c}=\kappa / 2 \pi \gamma$ is the corner frequency, $D=k_{B} T / \gamma$ is the diffusion coefficient, $\kappa$ is the stiffness constant of the trap in the given direction, $\gamma$ is the friction coefficient of the particle, $T$ is the temperature of the sample, and $k_{B}$ is the Boltzmann constant. At each position of the optical trap the PSD of the acquired time series (sampling frequency $f_{s}=4096 \mathrm{~Hz}$, number of samples $N_{s}=100000$ ) was calculated and fitted to the theoretical Lorentzian shape, getting the value for the calibration parameters [20-23].

This method depends upon the precise knowledge of the viscous drag coefficient, and hence the friction coefficient of the particle, the estimate of which is prone to large error when close to a surface, as in our experiment. Its dependence on the ratio between the particle size and distance to the surface must be accounted for to calibrate accurately the probe displacement. It is taken into account following the methodology proposed in [21], which relies on the expression of the diffusion coefficient for a sphere near a surface [24]. The validity of this formula for experiments similar to ours was confirmed in [25]. The uncertainty in the determination of the distance between the particle and the surface and hence the value of the diffusion coefficient is the leading source of error in the absolute value of the measured force. For example, for a $2 \mu \mathrm{m}$ particle placed at $500 \mathrm{~nm}$ from the surface, the relative error is estimated to be around $12 \%$.

An additional difficulty in the use of a PFM near a metal surface is the reflection of the trapping beam from the surface $[4,9,26]$. The reflected wave acts as an additional external force that changes the equilibrium position of the optical trap. This force is accounted for by calibrating the optical trap independently for each position of the probe.

The sphere moves towards a new equilibrium position under the effect of the plasmon radiation forces, which can be calculated along each coordinate axis from the knowledge of the displacement and the stiffness of the trap. In practice, two potential profiles are reconstructed with and without the SP field and their difference gives the SP radiation force [9]. Other interactions related, for example, to the gravitational force and the interaction with the surface can be eliminated. In each experiment we use a unique chamber with three types of probe particles so that all changes of measured radiation forces may be mainly assigned to the changes in momentum transfer, and not to the changes of setup geometry. In the micron size range the stiffness grows with the decrease of the probe size (see, for example, [27]). Hence, measurements done with probes of different sizes permit one to gain information on radiation forces using the PFM with different sensitivity. The stiffnesses of the optical trap along the off-axis $(x)$ direction measured far from the surface are $1.1 \mathrm{pN} / \mu \mathrm{m}(4.5 \mu \mathrm{m})$, $1.3 \mathrm{pN} / \mu \mathrm{m}(2.0 \mu \mathrm{m})$, and $1.8 \mathrm{pN} / \mu \mathrm{m}(0.6 \mu \mathrm{m})$. These values change considerably when approaching the surface.

A difficulty may arise due to the local increase of temperature at the metal layer produced by the SP excitation. A convection induced by such a local heater would produce a force at the probe in all three directions. However, we observe that the probe displacement occurs only along the $x$ and $z$ directions (Fig. 2) so we consider the local heating effects can be neglected when compared to radiation forces.

Previously, various publications reported the experimental analysis by PFM of the radiation pressure exerted on dielectric and metallic particles within a homogeneous evanescent field near a dielectric interface $[9,28]$. However, no studies have been done on radiation forces induced by SP.

Figure 2 shows typical potential energy profiles in the $x$ and $z$ directions with and without the SP wave when a $2 \mu \mathrm{m}$ bead is used as a probe. In this experiment, the incident angle is kept at resonance. When the distance between the probe and the surface is large enough, i.e., $\geq$ $500 \mathrm{~nm}$, both the $x$ potential [Fig. 2(a)] and the $z$ potential [Fig. 2(e)] can be well fitted to a parabolic function, and the plasmon radiation pressure results in a shift of the potential minima. When the probe is placed closer to the surface (the 

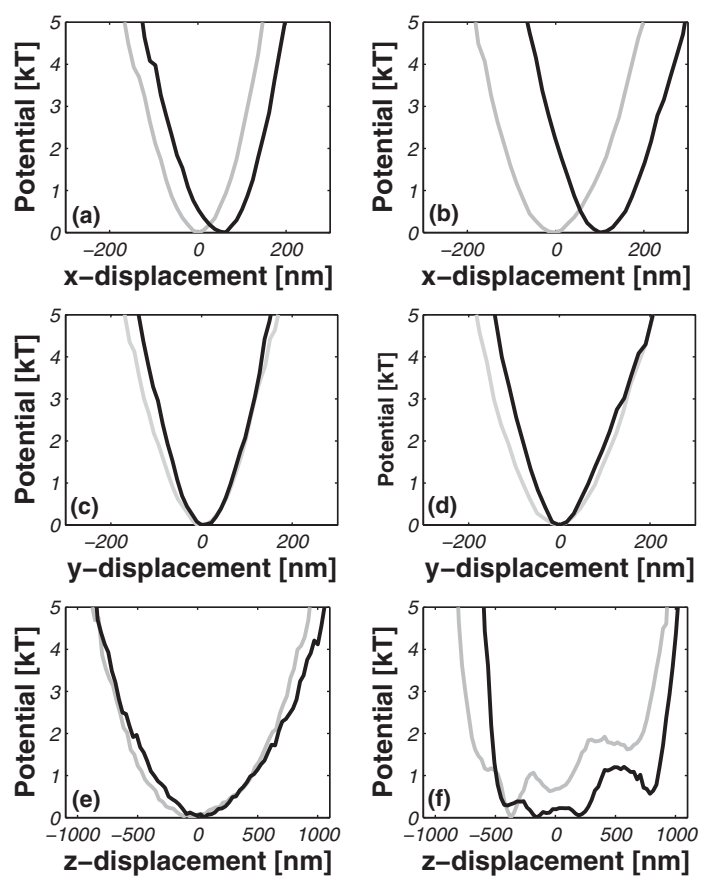

FIG. 2. Potential profiles along the $x, y$, and $z$ directions for a $2.0 \mu \mathrm{m}$ polystyrene bead placed at $500 \mathrm{~nm}[(\mathrm{a}),(\mathrm{c})$ and (e)] and

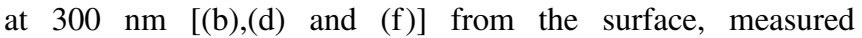
without (gray lines) and with (black lines) SP. No significative change is observed in the $y$-potential profile.

upper surface of the probe is around $300 \mathrm{~nm}$ from the metal) the potential profile along the $x$ direction [Fig. 2(b)] does not change significantly, while the one along the $z$ direction [Fig. 2(f)] reveals a more complicated behavior. This is attributed to the presence of additional forces of different natures exerted by the surface itself [2931] as well as due to the interference between the incident and reflected trapping beams. Multiple scattering between the surface and the probe of the evanescent field could also contribute to these oscillations [32]. However, because the oscillations in the potential are observed also when the laser exciting the plasmon field is off [Fig. 2(f)], we attribute them mainly to the reflection of the trapping beam. A similar behavior of the potential profiles has been observed with the three sizes of probes. In the following treatment, we analyze only radiation forces obtained from measurements at distances from the surface where the potential profiles measured are harmonic.

To distinguish the contributions of the incident electromagnetic wave and the resonant charge density oscillations to the total momentum of the SP transferred to a dielectric particle, we exploit the fact that the SP can only be coupled (i) at a specific incidence angle, and (ii) for a TM-polarized incident electromagnetic field. Hence, the respective contributions can be decoupled by changing either the angle or the polarization of the incident beam. These two cases are shown in Fig. 3(a), where the modulus of the plasmon radiation force acting on a $4.5 \mu \mathrm{m}$ polystyrene bead, placed at $500 \mathrm{~nm}$ from the metal surface, is plotted as a function of the incident angle. For the low-power optical trap we need, the $4.5 \mu \mathrm{m}$ sphere is found to be the most appropriate probe to guarantee the stability of the trapping through such a long-run experiment. A comparison with the plasmon coupling efficiency $(1-R$, where $R$ is the reflectivity) at the gold-water interface clearly shows that the plasmon radiation force follows the dispersion of the SP mode. This fact confirms that the presence of the particle located at such a distance does not significantly affect the SP. Also, by changing the polarization of the incident wave from TM to TE the force falls to a value similar to the one measured at a nonresonant angle. From these data it is possible to estimate the enhancement factor of the forces due to the SP (the angular interval of the resonance is very narrow so that we can neglect the variation of the penetration depth of the electromagnetic field inside this interval), which results in being about 40, in good agreement with the theoretical prediction (about 30) of electric field intensity increase at the gold-water interface. Further information is contained in the evolution of the plasmon radiation force vector with the incident angle [Fig. 3(b)]. The $z$ component at resonance is larger than the $x$ component. Theoretical predictions made for 10 times smaller dielectric particles show the same feature [17]. For an equal probe-surface distance we observe the same property also for a $0.6 \mu \mathrm{m}$ sphere, but the longitudinal force is found to be larger in the case of a $2.0 \mu \mathrm{m}$ one. For a full understanding of this experimental result, calculations are required for micrometer particles, since in this range the radiation force in an evanescent field is not expected to be monotonous as a function of their diameter [33].

The radiation force depends on the distance separating the surface and the particle, but also on the dimensions of

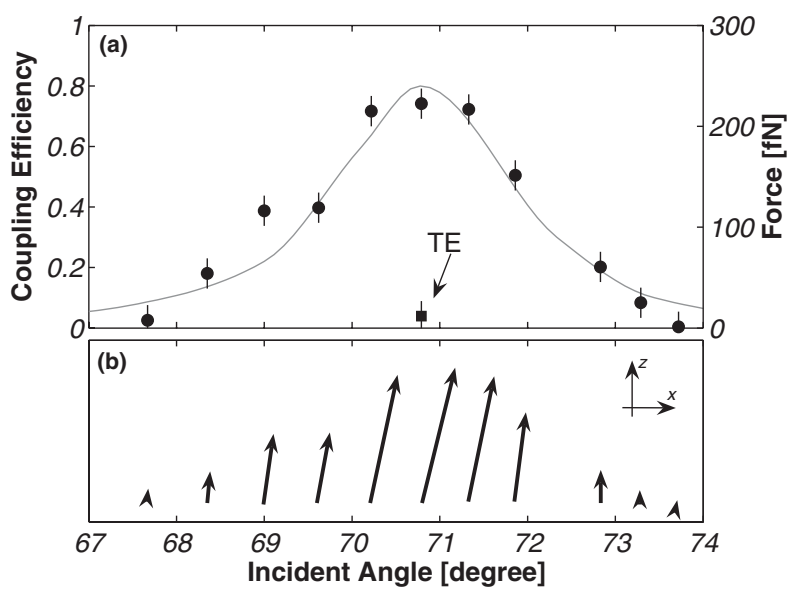

FIG. 3. (a) Coupling efficiency of the SP (continuous line) and modulus of the plasmon radiation force on a $4.5 \mu \mathrm{m}$ polystyrene bead under TM (dots) and TE (square) illumination. The bars represent the standard deviation of the measurement. (b) Evolution of the corresponding plasmon radiation force vectors vs the incident angle. 


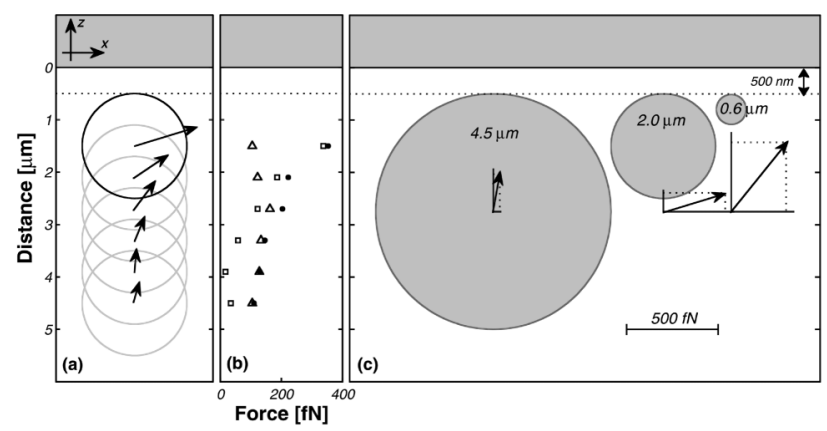

FIG. 4. (a) Force vector and (b) module (circles), $x$ component (squares), and $z$ component (triangles) of the force versus distance from the surface for a $2.0 \mu \mathrm{m}$ bead. (c) Force on beads of different sizes placed at $500 \mathrm{~nm}$ from the surface.

the particle itself. Figs. 4(a) and 2(b) illustrate the evolution of the radiation force vector acting on a $2 \mu \mathrm{m}$ particle with the distance from the metal surface. As expected, the modulus of the total force increases when the distance is reduced. The $z$ dependencies of the two components are different: the increase along the $x$ component is much sharper than the one along the $z$ component. Both of them do not follow the exponential tail of the electromagnetic field. The same experiment, repeated with 4.5 and $0.6 \mu \mathrm{m}$ beads, shows a similar tendency. For Rayleigh particle probes one would expect to observe an exponential $z$ decay of the force modulus following the exponential decay of the plasmon field. Indeed, in that case, the probe would act as a dipole which does not significantly modify the incident field and the force magnitude would then be determined by the incident field intensity at the probe position. In our experiments where the sizes of the probes are larger than the incident wavelength, the force magnitude results from a more complex process since the probe is big enough and the evanescent light can propagate inside it.

Figure 4(c) illustrates the force vector acting on the three kinds of particles, when the distance between the metal layer and the upper surface of all three particles is $500 \mathrm{~nm}$. The total force modulus increases as the probe size decreases, over the range of dimensions we consider. The absolute value of the plasmon force measured for a $0.6 \mu \mathrm{m}$ sphere is in reasonable agreement with the theoretical values [17]. Additionally, the force vector direction varies with the probe size. This effect can find application in optical sorting devices. A SP produced by a homogeneous metallic layer can act as a particle sorter since the velocity due to the plasmon radiation force of bigger particles will be considerably smaller than that of small particles: less force acting over more mass.

We believe that this study delivers an important contribution towards a better understanding and optimization of optical trapping forces produced by SP-enhanced evanescent waves, providing both a deeper understanding of the SP physics and an experimental approach to their implementation.

We thank Lluís Torner for helpful discussions and support. This research was carried out in the framework of ESF/PESC (Eurocores on Sons), through Grant No. 02-PESONS-063-NOMSAN, and with the financial support of the Spanish Ministry of Science and Technology. It was also partially supported by the Departament d'Universitats, Recerca i Societat de la Informació and the European Social Fund, and by the European Commission through Grant No. ATOM3D FP6-508952.

*Electronic address: Dmitri.Petrov@icfo.es

[1] H. J. Metcalf and P. van der Straten, Laser Cooling and Trapping (Springer, New York, 1999).

[2] A. Ashkin, Proc. Natl. Acad. Sci. U.S.A. 94, 4853 (1997).

[3] D. Grier, Nature (London) 424, 810 (2003).

[4] M. Nieto-Vesperinas et al., Phil. Trans. R. Soc. A 362, 719 (2004).

[5] C. Girard, Rep. Prog. Phys. 68, 1883 (2005).

[6] S. Kawata and T. Sugiura, Opt. Lett. 17, 772 (1992).

[7] S. Kawata and T. Tani, Opt. Lett. 21, 1768 (1996).

[8] P. C. Chaumet and M. Nieto-Vesperinas, Phys. Rev. B 62, 11185 (2000).

[9] K. Wada et al., Appl. Phys. Lett. 76, 2815 (2000).

[10] K. Sasaki et al., Opt. Lett. 25, 1385 (2000).

[11] V. Garcés-Chávez et al., Appl. Phys. Lett. 86, 031106 (2005).

[12] L. Novotny et al., Phys. Rev. Lett. 79, 645 (1997).

[13] R. Quidant et al., Opt. Lett. 30, 1009 (2005).

[14] H. Xu and M. Kall, Phys. Rev. Lett. 89, 246802 (2002).

[15] H. Raether, Surface Plasmons on Smooth and Rough Surfaces and on Gratings (Springer, New York, 1988).

[16] W. L. Barnes et al., Nature (London) 424, 824 (2003).

[17] Y. G. Song et al., Opt. Commun. 198, 7 (2001).

[18] W. Denk and W. W. Webb, Appl. Opt. 29, 2382 (1990).

[19] L. P. Ghislain and W. W. Webb, Opt. Lett. 18, 1678 (1993).

[20] A. Pralle et al., Microsc. Res. Tech. 44, 378 (1999).

[21] K. Berg-Sørensen and H. Flyvbjerg, Rev. Sci. Instrum. 75, 594 (2004).

[22] A. Rohrbach et al., Rev. Sci. Instrum. 75, 2197 (2004).

[23] A. Rohrbach, Phys. Rev. Lett. 95, 168102 (2005).

[24] J. Happel and H. Brenner, Low Reynolds Number Hydrodynamics (Springer, New York, 1983).

[25] A. Pralle et al., Appl. Phys. A 66, S71 (1998).

[26] P. Jákl et al., J. Mod. Opt. 50, 1615 (2003).

[27] A. Mazolli et al., Proc. R. Soc. A 459, 3021 (2003).

[28] A. R. Clapp and R. B. Dickinson, Langmuir 17, 2182 (2001).

[29] Y. Matsuo et al., J. Appl. Phys. 89, 5438 (2001).

[30] T. Ota et al., Appl. Phys. Lett. 80, 3448 (2002).

[31] K. Wada et al., Appl. Phys. Lett. 81, 1768 (2002).

[32] P. C. Chaumet and M. Nieto-Vesperinas, Phys. Rev. B 61, 14119 (2000).

[33] E. Almaas and I. Brevik, J. Opt. Soc. Am. B 12, 2429 (1995). 